Trauma Berufskrankh 2010 · 12[Suppl 2]:104-112 DOI 10.1007/s10039-010-1617-8

Online publiziert: 9. Mai 2010

(c) Springer-Verlag 2010
P. Biberthaler ${ }^{1}$ V. Bogner ${ }^{1} \cdot$ V. Braunstein ${ }^{1} \cdot$ S. Wirth ${ }^{2} \cdot$ W. Mutschler $^{1}$

${ }^{1}$ Chirurgische Klinik, Innenstadt, Ludwig-Maximilians-Universität München

${ }^{2}$ Institut für Klinische Radiologie, Innenstadt, Ludwig-Maximilians-Universität München

\title{
Radiuskopffrakturen bei Erwachsenen und Kindern
}

\section{Aktuelle Konzepte}

\section{Hintergrund}

Inzidenz. Radiuskopffrakturen des Erwachsenen sind relativ häufige Verletzungen (zwischen 1,5 und 4\% aller Frakturen) und betreffen $33 \%$ aller Ellenbogenfrakturen $[3,5,11,13]$.

Verletzungsursache und -mechanismus. Die Unfallursache ist in der Regel ein Sturz auf den leicht angewinkelten oder ausgestreckten Arm bei gleichzeitig gestrecktem Handgelenk (• Abb. 1): Das Individuum stürzt und versucht, über unbewusste Stellreflexe getriggert, die kine-

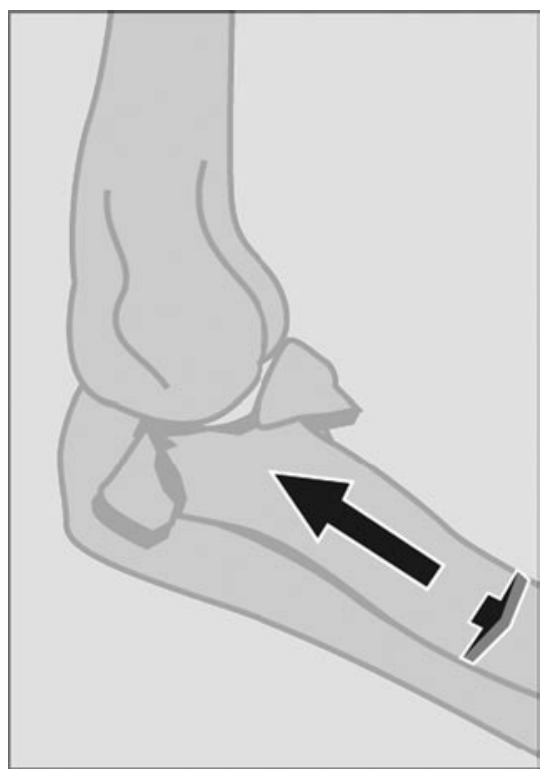

Abb. $1 \Delta$ Schema des typischen Unfallmechanismus bei Radiuskopffraktur, Pfeile Richtung der eingeleiteten Aufschlagsenergie (Zeichnung: Frau Hella Thun) tische Energie des Sturzes nicht auf den Kopf auftreffen zu lassen. Hierzu wird der Arm in mehr oder weniger ausgestreckter Haltung abgespreizt, um die Aufschlagsenergie abzufangen. Diese wird dann über das Handgelenk in den Unterarm eingeleitet (Pfeile in - Abb. 1) und trifft auf das Ellenbogengelenk. Je nachdem, ob dieses gestreckt gehalten oder in leichter Beugestellung straff fixiert wird, kann die Energie nicht über die Muskeln des Oberarms gepuffert werden, sondern kumuliert sich im Radiuskopf, der dann bricht.

Neben dislozierten, isolierten Radiuskopffrakturen wird, gerade bei gleichzeitig vorliegender Ellenbogenluxation, häufig auch das Auftreten von Radiuskopffrakturen in Kombination mit weiteren knöchernen Verletzungen (Processus coronoideus, Ulna usw.) oder Weichteilverletzungen, wie des medialen („medial collateral ligament“, MCL) sowie des lateralen Kollateralbandes („lateral collateral ligament“, LCL), oder als Kombinationsverletzung beobachtet (,unhappy triad „des Ellenbogens: Luxation, Radiuskopffraktur und Processus-coronoideusFraktur).

Versorgung. Während das Management von einfachen Frakturen in der internationalen Literatur relativ einheitlich beschrieben ist, liegen für komplexe Trümmerfrakturen des Radiuskopfes teilweise erheblich kontroverse Meinungen vor. Dabei favorisieren manche Autoren die offene Reposition und Osteosynthese („open reduction and internal fixation“, ORIF), während andere die primä- re Radiuskopfprothese und wieder andere die primäre Resektion des Radiuskopfes (Resektionsarthroplastik) bevorzugen und ihre Argumente mit teilweise widersprüchlichen Daten stützen [17]. In früheren Arbeiten wurde der Radiuskopf auch als entbehrlich beschrieben, jedoch weisen jüngere Untersuchungen ganz klar auf seine wesentliche Bedeutung als Stabilisator des Ellenbogens und der Unterarmgelenke hin $[9,16]$. In diesem Zusammenhang zeigte sich, dass die primäre Radiuskopfresektion mit Langzeitkomplikationen, wie beispielsweise Schmerzen, Gelenkinstabilität, proximale Translation des Radius, reduzierte Kraft im Unterarm, erhöhtes Osteoarthroserisiko und Valgusfehlstellungen im Ellenbogen, assoziiert sein kann $[11,16,23]$.

Entscheidend für das Management instabiler Luxationsfrakturen des Ellenbogens sowie von Ellenbogenverletzungen, welche die Membrana interossea (EssexLopresti-Verletzung) betreffen, ist die sorgfältige Rekonstruktion des Radioulnargelenks entweder durch Rekonstruktion des Radiuskopfes oder durch primäre Radiuskopfprothese [6]. In diesem $\mathrm{Zu}$ sammenhang wurde von einigen Autoren vorgeschlagen, dass beim gesunden aktiven jungen Patienten der primären Rekonstruktion gegenüber dem primären endoprothetischen Ersatz Vorzug gegeben werden sollte $[7,22]$. Diese Diskussion erfuhr in der jüngeren Vergangenheit durch die Entwicklung von schlankeren, weniger auftragenden Implantaten und schonenderen Operationstechniken einen leichten Trend in Richtung ORIF [17, 22, 
26]. Dieses Verfahren ist jedoch technisch anspruchsvoll und kann bei Komplikationen zu einem schlechten funktionellen Ergebnis führen. Kürzlich veröffentlichte Arbeiten versuchen zunehmend, sich mit Radiuskopfprothesen zu befassen, insbesondere nach Trümmerfrakturen des Radiuskopfes oder gleichzeitiger Instabilität des Ellenbogens bei MCL-Beteiligung [1, $2,13,20]$.

\section{Biomechanik des Radiuskopfes}

Der Radiuskopf stabilisiert den Ellenbogen und Unterarm im Wesentlichen in 2 Richtungen. Die radiohumerale Abstützung sorgt einerseits für eine Neutralisation von Valgusstresskräften, andererseits in longitudinaler Richtung dafür, dass der Unterarm, das distale Radioulnargelenk (DRUG) und das Handgelenk bei distaler Belastung abgestützt werden. Intraartikuläre Frakturen des Radiuskopfes reduzieren die Oberfläche, welche für die Krafteinleitung zur Verfügung steht, und damit die Ellenbogenstabilität [6, 25].

\section{Valgusstabilität}

Der Radiuskopf ist ein wesentlicher Stabilisator gegenüber Valgusstress des Ellenbogens, insbesondere wenn das MCL zerstört ist. Diese Situation tritt beispielsweise bei Luxationsfrakturen des Ellenbogens auf.

In einer Kadaverstudie zeigten Morrey et al. [23], dass der Radiuskopf als sekundärer Stabilisator gegenüber Valgusstress fungiert. Seine Retention führt zu einer Veränderung des Rotationszentrums der Varus-Valgus-Ebene, sodass der Hebelarm und damit die relative Belastung auf das MCL reduziert werden können. Laterale Ellenbogeninstabilitäten werden durch die Kombination einer Zerstörung des LCL („lateral collateral ligament“) und Druck auf das Radiohumeralgelenk begünstigt [15]. Bei Patienten mit akuter Instabilität ist die Rekonstruktion des LCL wichtiger als die des MCL. Dies geht aus Arbeiten hervor, die eine signifikante Abnahme der Ellenbogenstabilität bei Patienten nach primärer Radiuskopfexzision und gleichzeitig vorliegender LCLRuptur nachwiesen $[3,4]$. Obwohl sich die Ellenbogenstabilität nach sekundärer

Trauma Berufskrankh 2010 - 12[Suppl 2]:104-112 DOI 10.1007/s10039-010-1617-8

C) Springer-Verlag 2010

\section{P. Biberthaler · V. Bogner - V. Braunstein · S. Wirth · W. Mutschler Radiuskopffrakturen bei Erwachsenen und Kindern. Aktuelle Konzepte}

\section{Zusammenfassung}

Radiuskopffrakturen sind beim Erwachsenen häufige Verletzungen, deren Ausmaß von minimal dislozierten Brüchen bis zu Trümmerluxationsfrakturen reicht. Um die Funktion des Ellenbogengelenks möglichst gut wiederherzustellen, ist ein adäquates Management unerlässlich. Hierbei ist es wichtig, gezielt nach zusätzlichen knöchernen oder Weichteilverletzungen zu suchen, um diese ggf. in gleicher Sitzung zu versorgen. Seit Einführung von konfektionierten Low-Profil-Minifragmentimplantaten ist die Osteosynthese der Radiuskopffraktur eine sehr gute Option. Bei Frakturen, die aufgrund ihrer Trümmerzone eine sinnvolle Rekonstruktion des Radiuskopfes nicht erlauben, werden die primäre
Endoprothese oder die Resektion des Kopfes empfohlen, wobei bislang keine guten klinischen Studien zu diesen Techniken vorliegen. Radiuskopffrakturen bei Kindern sind aufgrund der späten Verknöcherung eher selten. Bei Kindern kommt es eher zu Radiushalsfrakturen, was die Durchblutung des Radiuskopfes bedrohen kann. Die Therapie erfolgt abhängig vom Alter des Kindes und vom Grad der Dislokation und ist eher konservativ ausgerichtet.

\section{Schlüsselwörter}

Radiuskopffrakturen · Erwachsene · Kinder . Ellenbogengelenkfunktion .

Begleitverletzungen

\section{Radius head fractures in adults and children. Current concepts}

\section{Abstract}

Radius head fractures are common injuries in adults ranging from minimally dislocated breaks to comminuted luxation fractures. In order to restore the function of the elbow joint in the best possible way a suitable management is indispensable. It is important to search for concomitant bony or soft tissue injuries in order to treat these simultaneously. Since the introduction of low profile tailored mini-fragment implants, osteosynthesis of radius head fractures is a very good option. For fractures which do not permit an expedient reconstruction of the radius head due to the fragmented area, a primary prosthesis or re- section of the radius head is recommended but as yet no good clinical studies have been published for any techniques. Radius head fractures in children are rare due to late ossification of the radius head and are more likely to suffer from radius neck fractures which can then affect the blood flow in the head. Therapy is dependent on the age of the children and the degree of dislocation and is more conservative.

\section{Keywords}

Radius head fractures · Adults - Children . Elbow joint function . Concomitant injuries 


\section{Ellenbogenverletzungen}

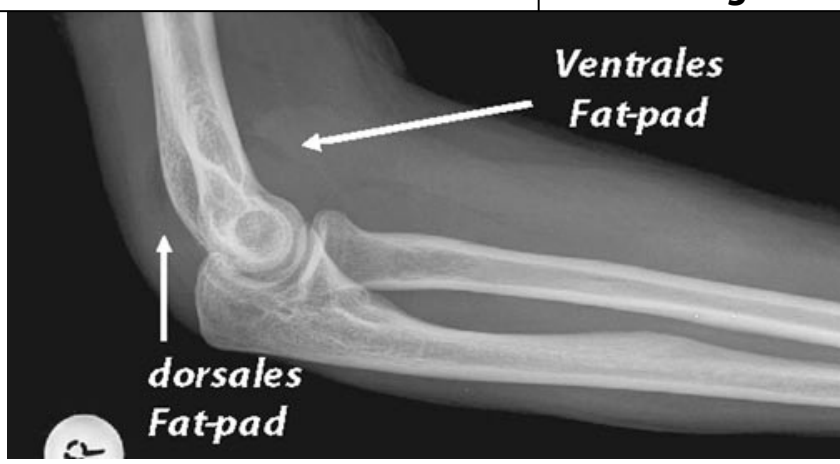

Abb. $2<$ Konventionelle Röntgenaufnahme des Ellenbogens im lateralen Strahlengang mit deutlich zu erkennendem ventralem und dorsalem „fat-pad sign" (Pfeile) als indirekte Frakturzeichen
Implantation einer Radiuskopfendoprothese steigern ließ, fanden sich diese Ellenbogen dennoch weiterhin relativ instabil im Vergleich zu Ellenbogen mit intakten ligamentären Strukturen. Diese Befunde legen nahe, dass die Rekonstruktion des LCL nach Radiuskopfprothese für die Ellenbogenstabilität essenziell ist $[3,4]$.

\section{Longitudinale Stabilität}

Das Ausmaß der Belastung zwischen Radius und Ulna hinsichtlich der Druckund Zugbelastung ist nicht eindeutig definiert. In jüngsten Veröffentlichungen wurde berichtet, dass der Radius bis $\mathrm{zu}$ 80\% der Belastung des Handgelenks aufnimmt, sich diese jedoch bis zum Ellenbogen gegenüber der Ulna auf die Hälfte ausgleicht $[14,27]$.

Morrey et al. [23] untersuchten Veränderungen der Kraftübertragung bei unterschiedlichen Unterarmrotationsstellungen und fanden, dass die größte Kraftübertragung vom Handgelenk in den Radiuskopf bei gestrecktem Ellenbogen und Unterarmpronation auftrat. Somit sind der intakte Radiuskopf und dessen intakte Artikulation mit dem distalen Capitulum humeri das primäre Gegenlager für die proximale Bewegung des Radius. Darüber hinaus wird dieser noch durch ligamentäre Strukturen stabilisiert, welche zusätzlich longitudinale Stabilität erbringen. Hierzu zählen die Membrana interossea des Unterarms sowie die Ligamente des DRUG (distales Radioulnargelenk). Nach Exzision des Radiuskopfes ist die physiologische Lastübertragung im Radiohumeralgelenk nicht länger gegeben, und die gesamten Druckkräfte von distal werden über die Ulna durch die Membrana interossea und die distalen Radioulnarbänder getragen [20]. Daher ist ein longitudinaler
Vorschub des Radius gegenüber der Ulna nach proximal zu erwarten, wenn nach Radiuskopffraktur zusätzlich eine Zerstörung einer dieser Stabilisatoren, insbesondere der Membrana interossea (EssexLopresti-Verletzung), vorliegt $[6,25]$.

\section{Erwachsene}

\section{Klinische und radiologische Untersuchung}

Bei Patienten nach Ellenbogentrauma und Radiuskopffrakturen ist der Ellenbogen deutlich geschwollen und bewegungseingeschränkt. Umso bedeutender ist die Dokumentation des Bewegungsumfangs sowohl der Flexions-Extensions- als auch der Pronations- und Supinationsmöglichkeiten nach der Neutralo-Methode. Darüber hinaus muss die Ellenbogenstabilität in Valgus- und Varusrichtung sowie hinsichtlich der a.-p. Luxationsneigung beurteilt und dokumentiert werden.

Radiologisch sollte der Ellenbogen mindestens in 2 Ebenen abgebildet werden, darüber hinaus kann bei Verdacht auf Radiuskopffrakturen eine GreenspanZielaufnahme des Radiuskopfes vorgenommen werden [8]. Die Computertomographie sollte bei unklaren Befunden oder indirekten radiographischen oder klinischen Frakturzeichen wie der intraartikulären Ergussbildung großzügig eingesetzt werden. Radiographisch äußert sich Letztere in der seitlichen Projektion durch die Verlagerung des ventralen "fat-pad“ sowie in der Erkennbarkeit des sonst nicht sichtbaren dorsalen „fat-pad“ (• Abb. 2). Diese Silhouette kommt durch eine weite Abhebung der Kapsel vom Knochen, die durch den intraartikulären Erguss verursacht wird, zustande. Die Computertomo- graphie kann damit auch wesentlicher Teil der therapeutischen Planung sein.

Für die Therapieplanung sollte die Verletzung zunächst klassifiziert werden. Bei Radiuskopffrakturen kommt die Einteilung nach Mason in Typ I-IV (• Abb. 3, - Tab. 1) in ihrer Modifikation nach Hotchkiss [14], zur Anwendung.

\section{Therapie}

\section{Wahl des Verfahrens}

Frakturen Typ Mason I werden frühfunktionell behandelt, d. h. ggf. Punktion (- Abb.4) eines bewegungseinschränkenden Hämarthros des Ellenbogengelenks (Entscheidungskriterium Streckhemmung bei $90^{\circ}$ ) und im Anschluss frühfunktionelle Physiotherapie sowie Kontrollröntgenuntersuchung nach 7 Tagen. Die anatomischen Landmarken zur Orientierung und Auffindung des Punktionsortes des Ellenbogengelenks sind in - Abb. 4 dargestellt. Ausgehend vom Olekranon lassen sich der laterale Epikondylus und der Radiuskopf gut tasten und ggf. mittels Fettstift markieren. Zur Sicherheit sollten immer nach medial der Sulcus nervi ulnaris mit seinem Nerven palpiert und der mediale Epikondylus markiert werden. Der optimale und ungefährlichste Ort für eine Punktion ist der Soft Spot zwischen Olekranon und Radiuskopf.

Bleibt die Verletzung undisloziert (Mason I), kann konservativ verfahren werden, ansonsten muss die operative Therapie erwogen werden (s. Mason II).

Radiuskopffrakturen Typ Mason II mit Dislokation um mehr als 2 mm oder jeglicher Gelenkverschiebung sowie die Frakturtypen Mason III und IV sollten offen rekonstruiert und mittels Osteosynthese bzw. primärer Radiuskopfprothese versorgt werden [30]. Prinzipiell steht noch die primäre Resektionsarthroplastik zur Verfügung. Kritisch bei der Versorgung dieser Frakturen kann die Einschätzung des Ausmaßes der Ellenbogeninstabilität sein. Radiuskopftrümmerfrakturen bedingen häufig posttraumatische Ellenbogeninstabilitäten, somit sollte das Management dieser Verletzungen immer auch die Ellenbogenstabilität adressieren. Deshalb ist es sehr wichtig, dass der behandelnde Chirurg den Zusammenhang 
Abb. 3 $>$ Klassifikation der Radiuskopffrakturen nach Mason, modifiziert nach Hotchkiss [14]

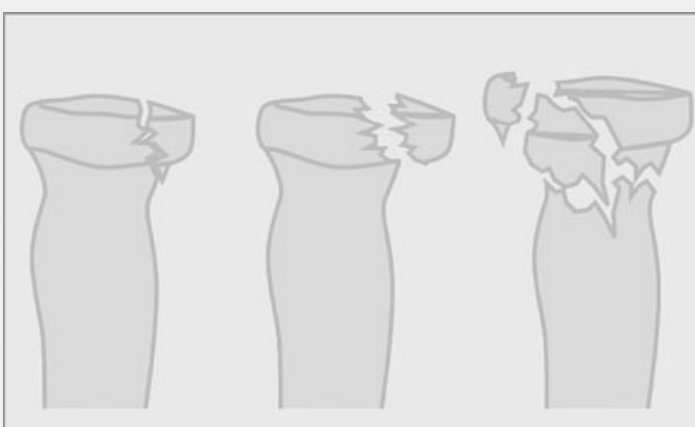

॥

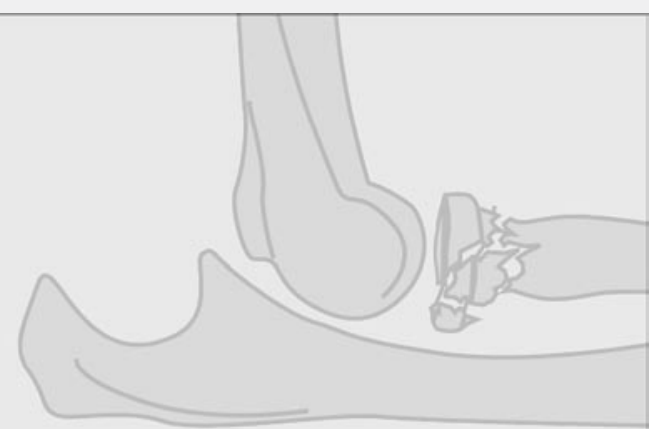

IV

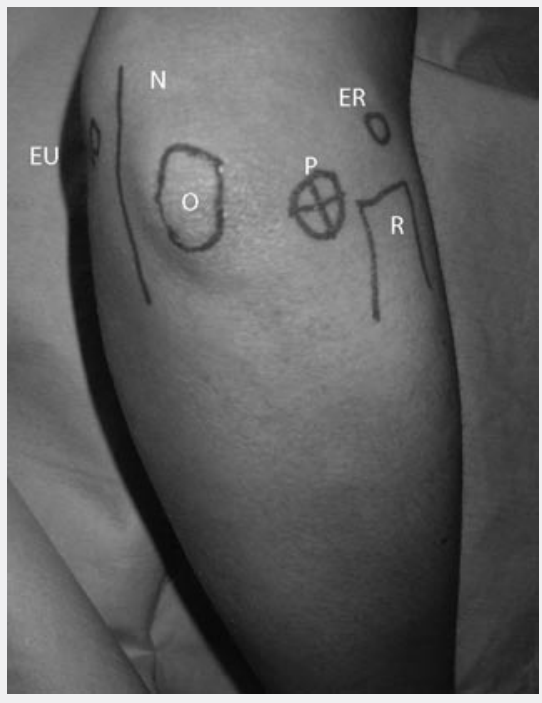

Abb. $4 \Delta$ Anatomische Landmarken zur Orientierung und Auffindung des Punktionsortes des Ellenbogengelenks, ER Epicondylus radialis, EU Epicondylus ulnaris, $N$ N. ulnaris, $O$ Olekranon, $R$ Radiuskopf, Kreuz Soft Spot zwischen Olekranon und Radiuskopf

Abb. 6 - Schematische Darstellung der optimaIen Position von Low-Profil-Implantaten auf der Zirkumferenz des Radiuskopfes in der "safe zone" (Zeichnung: Frau Hella Thun)

zwischen den bestehenden ossären und ligamentären Verletzungen versteht und die notwendigen Maßnahmen ergreift, diesen entgegenzuwirken. Dabei hat beispielsweise die Refixierung eines potenziell dislozierten Processus coronoideus deutliche Auswirkungen auf die Ellenbogeninstabilität. Kleine Typ-I- oder Typ-II-Koronoidfrakturen lassen sich ggf. mittels Naht re-
Abb. 5 Schematische Darstellung des eignen chirurgischen Zugangs etwas weiter ulnaseitig als der Standard-Kocher-Zugang zwischen M. extensor carpi ulnaris und M. extensor digitorum

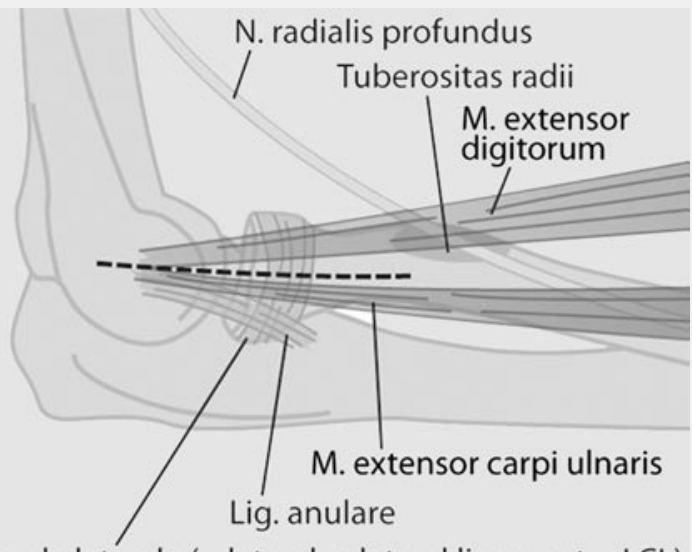

Lig. collaterale laterale $(=$ lateral colateral ligament $=\mathrm{LCL}$ )

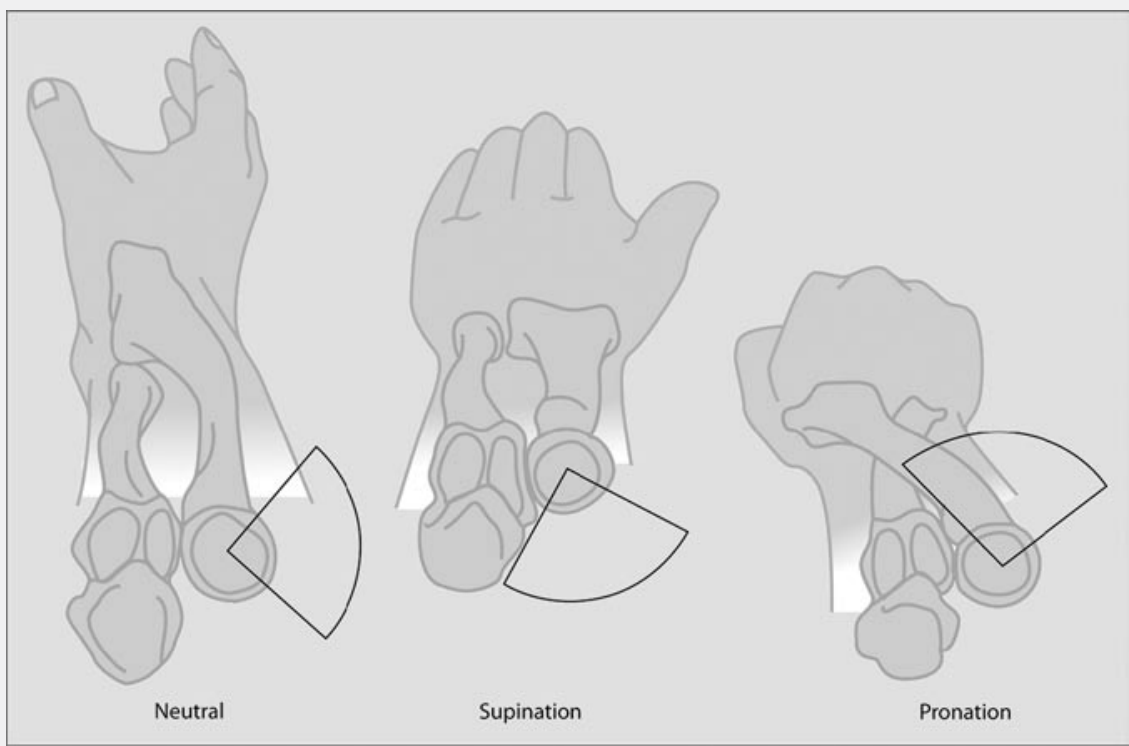

konstruieren, während größere Typ-IIIFrakturen mittels Schrauben oder Platten fixiert werden können. Hierbei sollte der Zugang über medial erfolgen.

Eventuell vorliegende ligamentäre Verletzungen sollten gleichzeitig mit der Radiuskopffraktur rekonstruiert werden, um die Stabilität wiederherzustellen. Dabei kann die Rekonstruktion des LCL über den gleichen Zugang, über den auch die Radiuskopffraktur versorgt wird, durch primäre Naht oder zusätzliche Bohrlöcher durch den lateralen Epikondylus bzw. Knochenanker erfolgen. Die Rekonstruktion des MCL ist normalerweise nicht notwendig, solange sich der Ellenbogen nach der Rekonstruktion des Processus coronoideus bzw. des LCL als stabil 
Tab. 1 Frakturtypen nach Mason in der Modifikation nach Hotchkiss [14]

\begin{tabular}{ll} 
Typ & Charakteristika \\
I & Nicht dislozierte Fraktur \\
\hline II & Fraktur mit einer Dislokation $>2 \mathrm{~mm}$ \\
\hline III & $\begin{array}{l}\text { Trümmerfraktur mit mehr als } \\
2 \text { Fragmenten }\end{array}$ \\
\hline IV & $\begin{array}{l}\text { Fraktur mit zusätzlicher } \\
\text { Ellenbogenluxation }\end{array}$ \\
\hline
\end{tabular}

erweist. Für diejenigen Gelenke, die selbst nach Rekonstruktion der Knochen und Ligamente weiterhin instabil erscheinen, mag die Applikation eines Bewegungsfixateurs notwendig sein.

\section{Chirurgischer Zugang}

Der Zugang zum Radiuskopf nach Kocher beginnt am lateralen Epikondylus und zieht longitudinal nach distal. Nach Durchtrennung der Haut stellt sich ein Sehnenspiegel dar, der nach distal in 2 Muskelzüge ausläuft:

- radialseitig in den M. anconaeus und

- ulnaseitig in den M. extensor carpi ulnaris.

Zwischen diesen beiden Muskelzügen befindet sich eine Faszie, die scharf in longitudinaler Richtung durchtrennt wird. Nach unserer Erfahrung ist dieser Zugang bisweilen etwas zu „radial“, sodass wir etwa $0,5 \mathrm{~cm}$ weiter ulnawärts zwischen dem M. extensor carpi ulnaris und dem M. extensor digitorum eingehen (- Abb. 5). Dabei kann die Orientierung aufgrund von Weichteilschwellungen deutlich eingeschränkt sein, daher empfiehlt es sich immer, die Landmarken möglichst optimal zu markieren. In jedem Fall ist auf den motorischen Ast des N. radialis zu achten, indem eine Präparation weiter distal als bis zur Tuberositas vermieden wird. Falls diese aus operationstechnischen Gründen doch notwendig sein sollte, muss ein weiter radial gelegener Zugang gewählt werden. Beiden Zugängen, dem Standard-Kocher-Zugang und unserer Modifikation, gemeinsam ist, dass ggf. darunter liegende Ausläufer des M. supinator longitudinal gespalten werden können. Dann sollte sich die Kapsel darstellen, die ebenfalls in Längsrichtung scharf eröffnet wird. Insgesamt sollte der Muskel-Kapsel-Komplex soweit mobilisiert werden, dass der LCL-Komplex eingesehen werden kann. Hierbei ist es wichtig, den motorischen Ast des N. radialis durch Pronation im Vorarm zu schützen. Durch diese Bewegung wird der Nerv vom Radiushals weg bewegt. Bei intakter Kapsel wird diese anteriorseitig des lateralen Kollateralligaments longitudinal inzidiert, und der anteriore Kapselanteil vom lateralen Epikondylus mobilisiert. Somit lässt sich der anterolaterale Anteil des Gelenks ohne Verletzung des lateralen Kollateralligaments darstellen. Dieser Zugang erlaubt eine gute Darstellung des Radiuskopfs, und die Rekonstruktion bzw. Osteosynthese können über ihn gut durchgeführt werden.

Sollte eine weiter distal gelegene Mobilisation notwendig sein, kann das Lig. anulare z-förmig inzidiert werden. Diese Technik wird in unserem Haus nicht durchgeführt, ist aber in den USA verbreitet. Hierdurch lässt sich ein stabiles Lig. anulare nach Rekonstruktion gewährleisten. Bei veralteten Verletzungen in fixierter Flexion kann versucht werden, durch ein anteriores Kapselrelease die Beweglichkeit des Ellenbogens zu verbessern.

\section{Chirurgische Technik}

ORIF. Bei 3 oder weniger Fragmenten ist die ORIF die bevorzugte Therapie. Hierfür sind folgende operative Instrumente notwendig:

- Kleine Kirschner-Drähte,

- Minifragmentfixierungsset (1,5- bzw. 2,o-mm-Schrauben) oder HerbertMinischrauben und

- Minifragmentradiusplättchen.

Die Gelenkfläche des Radiuskopfes ist dabei unter Sicht zu reponieren und passager mittels Kirschner-Draht zu fixieren.

Nachdem die korrekte Reposition im Bildwandler dokumentiert wurde, erfolgt die definitive Fixierung entweder mittels 1,5- oder 2,0-mm-Minifragmentschrauben. Wichtig ist dabei, den Schraubenkopf in jedem Fall unter das Knorpelniveau der Zirkumferenz versenken, um Störungen bei Pronation-Supinations-Bewegungen zu vermeiden.

Frakturen die über den Radiuskopf in den Radiushals hinausreichen, erfordern die Fixierung mittels Miniplattenosteosynthese. Dabei sollte die Platte in der so genannten „safe zone“ platziert werden (- Abb. 6). Sie entspricht der Oberfläche des Radiuskopfes, welche nicht mit dem proximalen Radioulnargelenk artikuliert und diesen nicht bei Unterarm- und Drehbewegungen berührt. Hierfür steht ein etwa 45 Bogengrad messender Anteil des Radiuskopfes zur Verfügung, der einfach durch Unterarmdrehbewegung nach temporärer Fixierung mit KirschnerDrähten fixiert werden kann. Dabei stellt die Tuberositas radii die distale Grenze der Plattenauflagefläche dar (• Abb. 7). Eine Plattenlage distal davon gefährdet den tiefen Ast des N. radialis erheblich.

Die typische Fehlreposition führt zu einer Valgusfehlstellung und Verkürzung des Radius. Daher sollte die Operation nur begonnen werden, wenn auch eine Radiuskopfprothese zur Verfügung steht, um bei ausgedehnten Trümmerfrakturen den primären endoprothetischen Ersatz des Radiuskopfes vornehmen zu können.

Primäre Resektionsarthroplastik. Sollte der Radiuskopf derart zertrümmert oder disloziert sein, dass eine ORIF keine Aussicht auf Erfolg hat, kann die primäre Resektion des Radiuskopfes erwogen werden. Diese Operationsmethode wird kontrovers diskutiert, über die Langzeitergebnisse liegen widersprüchliche Studien vor $[11,16]$. Herbertsson et al. [11] berichteten über sehr erfolgreiche Ergebnisse mit zufrieden stellenden Outcomes für 54 von 61 Patienten in einer Langzeituntersuchung nach Radiuskopfresektion.

Die Radiuskopfresektion ohne primäre Prothese ist bei begleitender Ruptur des medialen Kollateralligaments (MCL) oder der interossären Membran (Essex-Lopresti-Verletzung) kontraindiziert. Aber auch ohne zusätzliche Weichteilverletzungen (z. B. Ruptur des MCL) kann sie mit Spätkomplikationen vergesellschaftet sein, wie Schmerzen, Gelenkinstabilität, proximale Radiustranslation, abgeschwächte Kraft, Osteoarthrose und Valgusfehlstellungen im Ellenbogen [16, 23].

Primäre Radiuskopfprothese. Sie ist bei dislozierter Trümmerfraktur des Radiuskopfes indiziert, wenn eine stabile Osteosynthese nicht möglich ist und die Fraktur mehr als die Hälfte des Radiuskopfes 
einnimmt ([2, 19], • Abb. 8). Insbesondere ist sie bei Frakturen mit zusätzlichen ligamentären Verletzungen (Ellenbogenluxationen oder distale Radioulnarverletzungen bei gleichzeitig bestehender Mason-III- und -IV-Fraktur) oder zusätzlichen Frakturen beispielsweise des Processus coronoideus oder einer Olekranonfraktur, welche disloziert oder instabil ist, indiziert. Die Kontroversen in der Literatur beziehen sich im Wesentlichen auf die Frage, welche Verletzungen exakt diese Kriterien erfüllen.

\section{Technische Überlegungen}

Die metallische Endoprothese soll den Radiuskopf so gut wie möglich ersetzen. Zahlreiche biomechanische Studien ergaben, dass hierbei die optimale Größe der Prothese wichtig ist $[24,25,27,28,31]$. Eine Prothese mit einem zu großen oder zu kleinen Durchmesser könnte zu einer unphysiologischen Belastung im Ellenbogengelenk führen. Darüber hinaus kann ein nicht optimaler Durchmesser des Radiuskopfes Druckeffekte auf das Capitulum des Humerus auslösen [32]. Der korrekte Durchmesser der Radiuskopfprothese ist durch einfaches Ausmessen des entfernten Radiuskopfes mit der Probierprothese relativ einfach zu ermitteln. Die Prothese sollte kongruent im Gelenk liegen und einen flüssigen Bewegungsablauf der Radiuskopfprothese mit dem Capitulum über den gesamten Bewegungsumfang erlauben $[2,21]$.

Darüber hinaus ist auch die Prothesenlänge von Bedeutung. Die meisten Frakturen des Radiuskopfes treten an dessen Übergang zum Radiushals auf, somit kann der korrekte Sitz der Prothese intraoperativ gut kontrolliert werden. Dabei ist darauf zu achten, dass der Radiushals in entsprechend korrekter Position abgesetzt werden kann, um eine stabile Verankerung der Prothese und einen sicheren Sitz zu gewährleisten. Hierbei ist darauf zu achten, dass der Radiuskopf gut mit dem Capitulum artikuliert, aber das Gelenk nicht in seinem Bewegungsumfang eingeschränkt ist.

\section{Postoperative Nachbehandlung}

Sie stellt einen wesentlichen Faktor im Management von Patienten nach Radiuskopffrakturen dar. Hierbei muss versucht
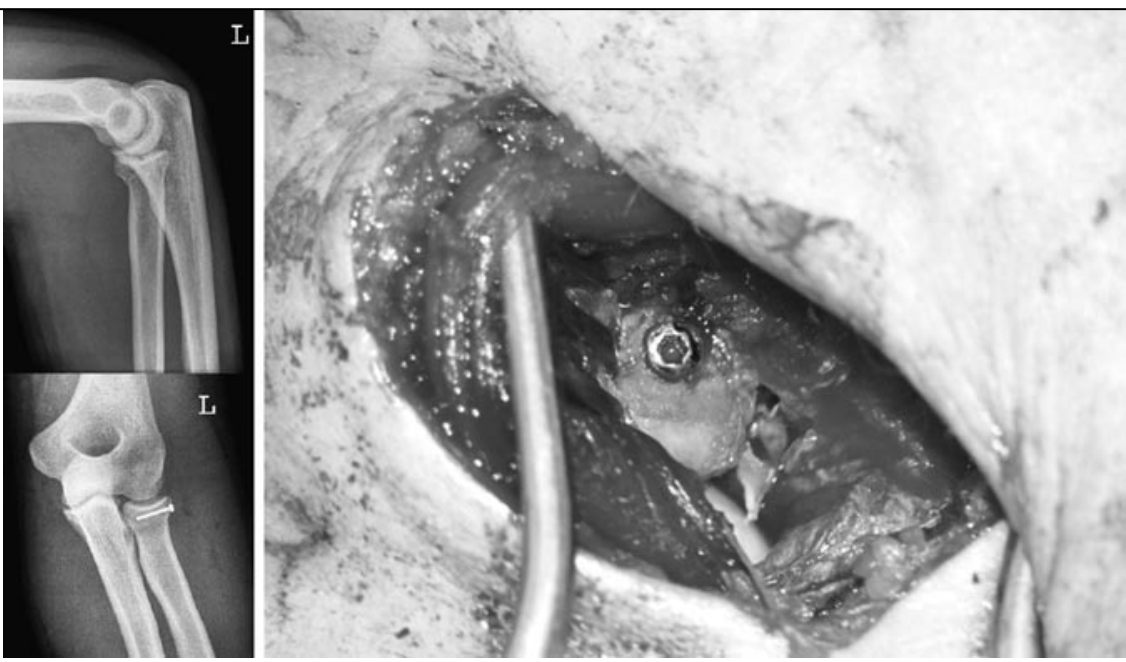

Abb. 7 \ Typische Mason-II-Fraktur (links oben) mit Dislokation um mehr als $2 \mathrm{~mm}$ und intraoperativer Situs nach Minifragmentschraubenosteosynthese (rechts) mit komplett im Knorpel der Zirkumferenz versenktem Schraubenkopf (Vermeidung einer Störung der Drehbewegungen des Radiuskopfes); Radiushalsfraktur mit Abkippung nach ORIF und Low-Profil-Miniplattenosteosynthese (links unten) mit deutlich zu erkennendem aufgerichtetem Hals sowie in der "safe-zone“" positionierter Platte
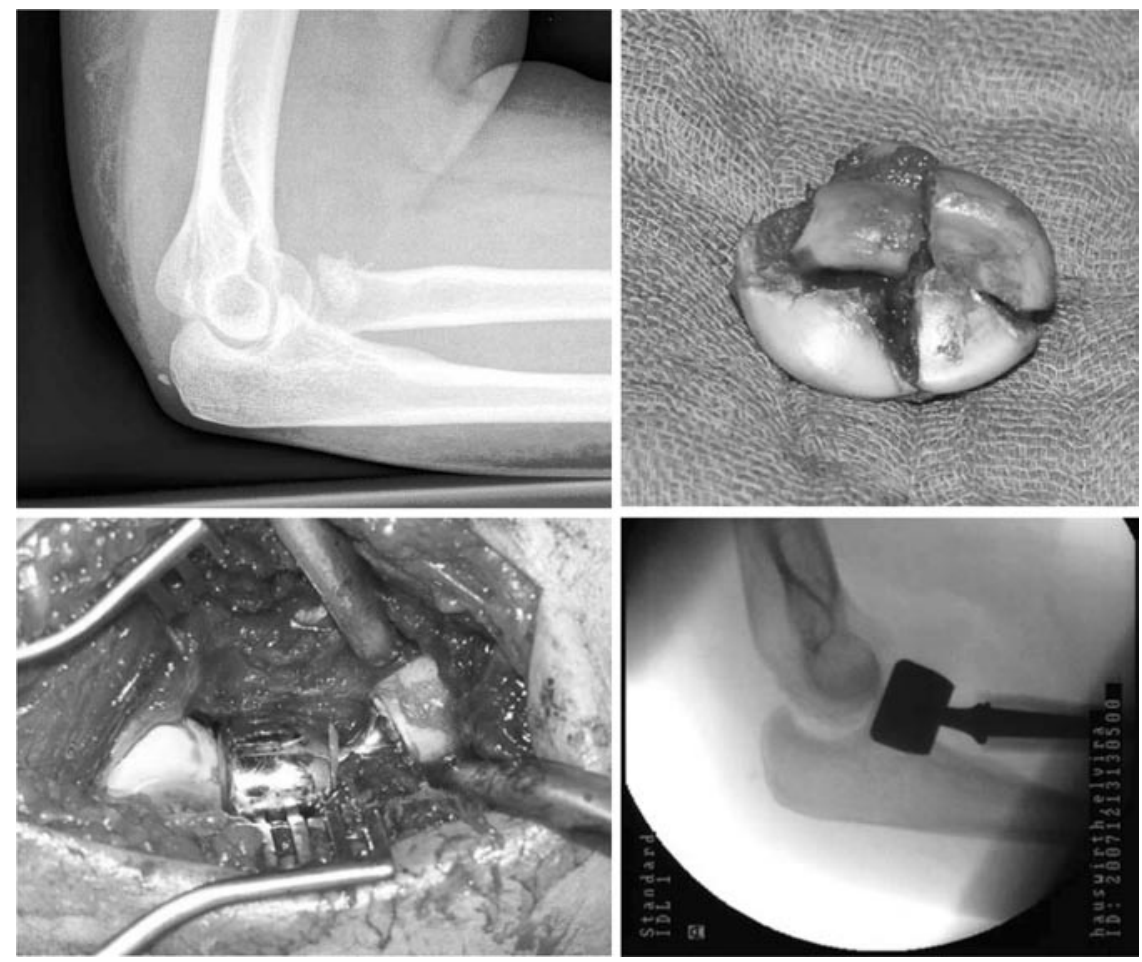

Abb. 8 \ Typische Radiuskopftrümmerfraktur Mason III, links oben konventionelles Röntgenbild, rechts oben intraoperativer Situs eines komplett abgeschlagenen Radiushalses und in mehreren Fragmenten liegende Zirkumferenz des Kopfes, links unten Radiuskopfprothese in situ mit leicht nach anterior gewinkelter, der physiologischen Orientierung des Radiuskopfes nachempfundener Neigung des Kopfes, rechts unten intraoperative Röntgenkontrolle

werden, auf der einen Seite die Instabilität zu berücksichtigen und auf der anderen Seite durch frühe Mobilisation die Beweglichkeit des Gelenks so optimal wie möglich zu erhalten. Daher sollte eine frühe Bewegung innerhalb eines sicheren Radi- us unter physiotherapeutischer Anleitung geübt werden.

Zur Vermeidung von heterotopen Ossifikationen ist die Applikation von Indomethacin als positiv beschrieben [24]. Eine weitere Therapieoption wird der Strahlentherapie zugesprochen. Stein et al. [29] 


\section{Ellenbogenverletzungen}

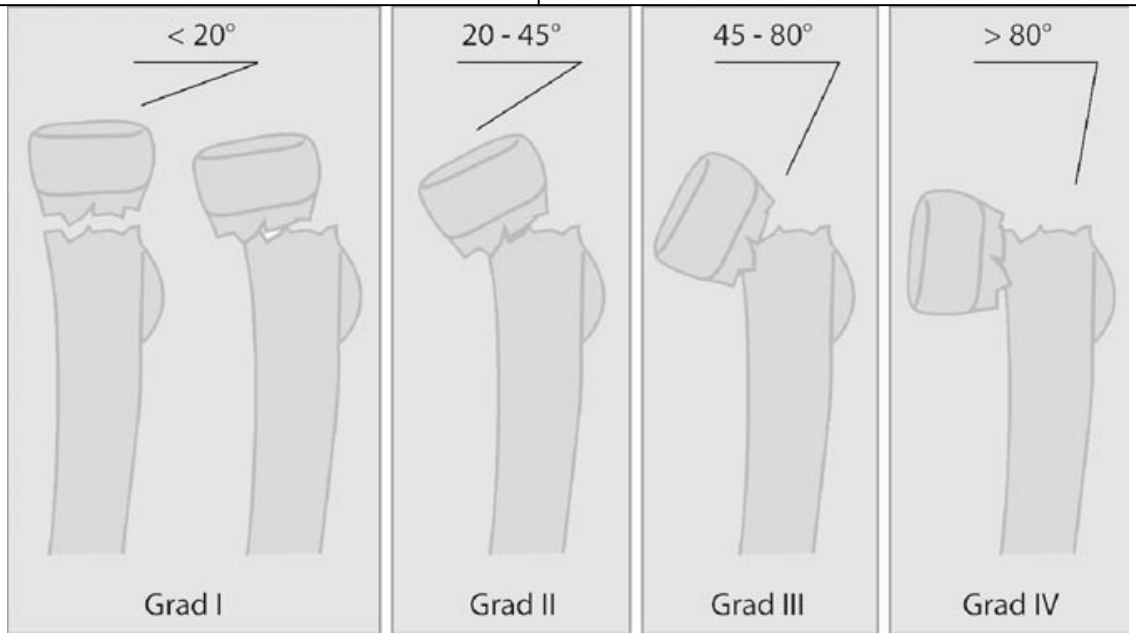

Abb. $9 \Delta$ Fraktureinteilung der kindlichen Radiuskopf-/-halsfrakturen nach Maitezeau in Abhängigkeit vom Grad der Abkippung des Kopffragmentes gegenüber dem Hals

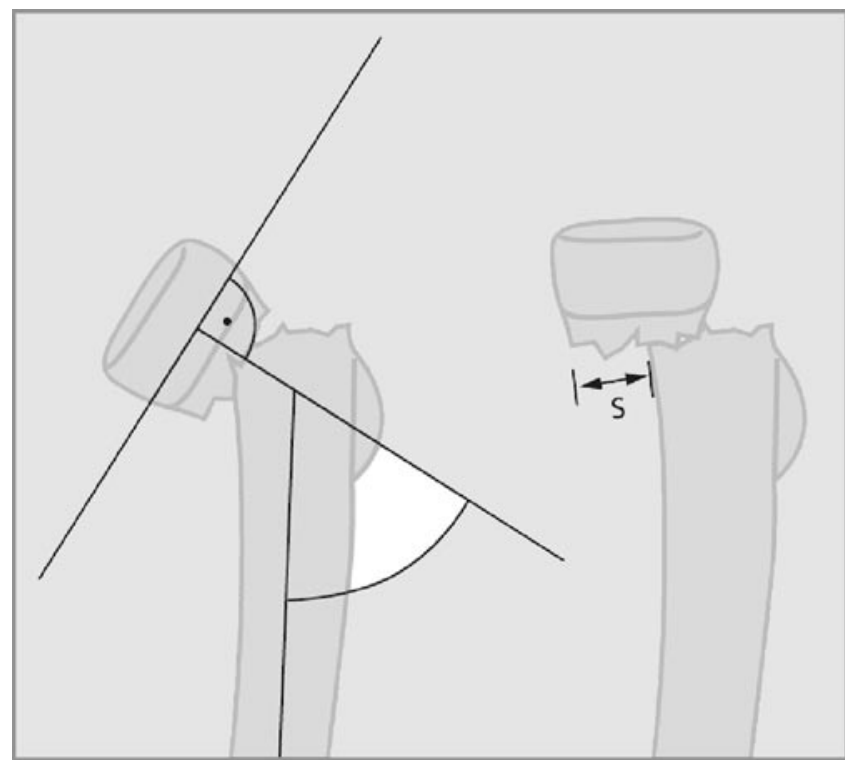

Abb. $10<$ Kriterien zur operativen Intervention kindlicher Radiuskopffrakturen berichteten über gute Ergebnisse bei 10 von 11 Patienten nach Ellenbogentrauma, welche mittels $700 \mathrm{cGy}$ Bestrahlung innerhalb von $72 \mathrm{~h}$ nach der Operation behandelt wurden.

\section{Ergebnisse}

Die aktuelle Literatur über die Versorgung komplexer Radiuskopf- und Halsfrakturen ist nicht einheitlich und erlaubt nur eine bestimmte Orientierung für die Auswahl des optimalen Managements, da die klinische Studienlage über die Behandlung von Radiuskopffrakturen mittels ORIF, Resektionen oder primärer Prothese zu heterogen ist, um daraus einen allgemeinen Behandlungsalgorithmus ableiten zu können $[16,17]$. Die Bandbreite der zusätzlich assoziierten Weichteilverletzungen des Ellenbogens erschwert die Beurteilung ein- zelner Effekte bzw. therapeutischer Regimes von Radiuskopffrakturen zusätzlich.

Ikeda et al. [18] verglichen die Ergebnisse von Radiuskopfresektionen mit denen der Osteosynthese in einer Serie von 28 Patienten. Zum Zeitpunkt der endgültigen Nachuntersuchung hatten die Patienten nach Osteosynthese eine bessere Kraft, Funktion und Gelenkbeweglichkeit im Gegensatz zu den Patienten nach Resektion. Bei der Betrachtung dieser Ergebnisse muss berücksichtigt werden, dass das Nachuntersuchungsintervall der Osteosynthese bei 3 Jahren lag, verglichen mit einem 10 Jahres-Follow-up der Resektionsgruppe.

Herbertsson et al. $[11,12]$ berichteten über ein Langzeitnachuntersuchungsintervall von durchschnittlich 18 Jahren und fanden nach Radiuskopfresektion gute Ergebnisse bei Mason-II- und -IIIFrakturen. Jedoch fanden sich im Vergleich zum nicht verletzten Ellenbogen eine deutlich erhöhte Häufung von posttraumatischer Arthrose ( $73 \%$ vs. $7 \%$ ).

King et al. [22] veröffentlichten $100 \%$ sehr gute Ergebnisse nach Osteosynthese nach Mason-II-Frakturen im Gegensatz zu $33 \%$ sehr guten Ergebnisse nach Mason-IIIVerletzungen und schlossen daraus, dass Trümmerfrakturen eher mittels primärer Prothese behandelt werden sollten.

Ikeda et al. [17] berichteten über sehr gute Ergebnisse von Mason-III- und -IVFrakturen nach Osteosynthese mittels konfektionierten Low-Profil-Minifragmentimplantaten. In dieser Serie von 10 Patienten wiesen 90\% gute bis sehr gute Ergebnisse auf.

In einer jüngsten Veröffentlichung beschrieben Ring et al. [26] die Versorgung von 56 Radiuskopffrakturen mittels Osteosynthese und kamen zu dem Schluss, dass diese nur für Frakturen mit 3 oder weniger artikulären Fragmenten geeignet sei. 13 von 14 Patienten nach MasonIII-Frakturen mit mehr als 3 artikulären Fragmenten hatten nicht zufrieden stellende Ergebnisse verglichen mit den zufrieden stellenden Ergebnissen bei $15 \mathrm{~Pa}$ tienten nach Mason-II-Fraktur. Bei den 12 Patienten mit einer Typ-III-Fraktur, bei denen der Radiuskopf in 1 oder 2 Fragmente gespalten war, trat kein frühes Implantatversagen auf, eine Patient wies eine "non-union“ auf und alle hatten eine Unterarmpronations-/-supinationsbewegung von mehr als $100^{\circ}$.

Bezüglich der primären Radiuskopfprothese wurde von Bain et al. [2] über 16 Patienten nach Mason-III-Verletzung und Versorgung mittels Monoblocktitanprothese berichtet. Die Autoren beschrieben $80 \%$ sehr gute Ergebnisse bei einem 2 1/2-jährigen Nachuntersuchungsintervall. Die wesentliche Schlussfolgerung dieser Arbeit war, dass die chirurgische Versorgung nicht verzögert werden soll und einer frühen Mobilisation wesentliche Bedeutung für ein zufrieden stellendes Ergebnis zukommt. Harrington et al. [10] berichteten über ähnliche Ergebnisse mit einem wesentlich längeren Nachuntersuchungsintervall von im Durchschnitt 12 Jahren nach primären Radiuskopfpro- 
Abb. 11 Minimalinvasive Versorgung einer kindlichen Radiuskopffraktur mittels intramedullärem Draht: links distale Eröffnung der Markraumhöhle und Einführen des Titandrahts in den Markraum unter Bildwandlerkontrolle, Mitte Vorschieben des Nagels bis zum abgekippten Fragment und Auffädelung desselben, rechts Aufrichtung des abgekippten Fragments durch Drehen am Nagel, Verspannen des Nagels

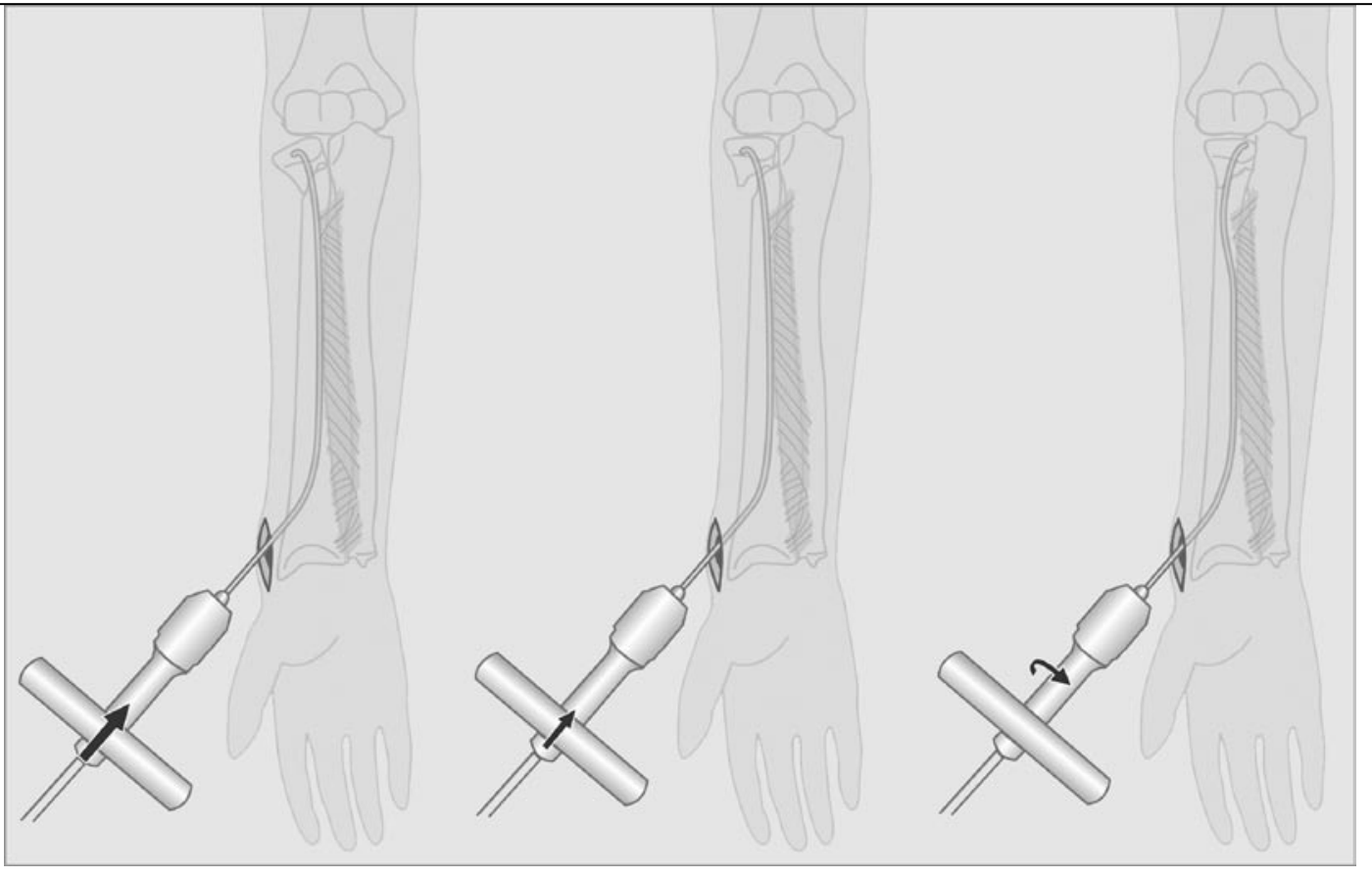

thesen nach instabilen Ellenbogenluxationsfrakturen.

Hinsichtlich der Versorgung mit modularen Implantaten liegen zum gegenwärtigen Zeitpunkt keine nennenswerten Arbeiten vor, die diese mit mono- oder bipolaren Radiuskopfprothesen verglichen. Die Problematik des Kopfdurchmessers der Radiuskopfprothese wurde in mehreren biomechanischen Studien gezeigt [32]. Allerdings liegen derzeit keine klinischen Berichte vor, dass diese Größenunterschiede tatsächlich auch klinisch relevante Differenzen in der Funktion bedingen. Darüber hinaus gibt es derzeit keine vergleichende prospektiv randomisierte klinische Studie zwischen ORIF, primärere Prothese oder Resektion.

\section{Radiuskopf- und -halsfrakturen bei Kindern}

\section{Verletzungsmechanismus und -folgen}

Der Unfallmechanismus ist wie beim Erwachsenen ein Sturz auf die ausgestreckte Hand. Frakturen des Radiuskopfes bzw. halses sind aufgrund der vor der Verknöcherung praktisch ausschließlichen Knorpelstruktur des Radiuskopfes eine Seltenheit (etwa 1\% aller Extremitätenfrakturen bei Kindern). Meißel- oder Trümmerfrakturen treten erst auf, wenn der Radiuskopf verknöchert ist. Somit sind Frak- turen des proximalen Radius fast ausschließlich metaphysäre Brüche, wobei 2/3 der Fälle metaphysäre Stauchungsfrakturen und etwa 1/3 Epiphysenlösungen mit bzw. ohne metaphysären Keil darstellen.

Die Blutversorgung des Radiuskopfes erfolgt über periostale Blutgefäße, sodass nach einer Fraktur des proximalen Radiusendes im Wachstumsalter Störungen der Blutversorgung zu Teil- oder Vollnekrosen des Radiuskopfs führen können.

\section{Klassifikation}

Proximale Radiusfrakturen werden nach Maitezeau Grad I-IV klassifiziert (• Abb. 9, 10). Dabei nimmt der Grad der Dislokation von Grad I bis Grad IV zu und ist somit Ausdruck einer wachsenden Gefährdung für die Blutversorgung, da diese im Wesentlichen über das Periost erfolgt, welches bei zunehmender Dislokation mit größerer Wahrscheinlichkeit rupturiert ist oder rupturgefährdet sein kann.

\section{Therapie}

\section{Ziel}

Das Therapieziel sind die Erhaltung bzw. Wiederherstellung der Funktion, sodass aufgrund der beschriebenen Durchblutungssituation die dislozierte Radiusköpfchenfraktur eine Notfallindikation in der
Kindertraumatologie darstellt. Für die Therapieentscheidung wesentlich sind zum einen der Abkippungswinkel, der als Epiphysenachsenwinkel ermittelt wird, zum anderen die Seit-zu-Seit-Verschiebung. Als akzeptable Dislokation gelten ungefähr die in $\mathbf{0}$ Tab. 2 aufgeführten Altersrichtwerte.

\section{Techniken}

Die geschlossene Reposition erfolgt im Wesentlichen durch Druck mit dem Daumen bei leicht gebeugtem Ellenbogen unter Pro- und Supinationsbewegungen auf den Radiuskopf. Ist sie nicht erfolgreich, kann die intramedulläre Markraumschienung durchgeführt werden. Hierbei wird am distalen Radius ein Titannagel (ESIN: elastische intramedulläre Stabilisierung) eingeführt, mit dessen gebogener Spitze der Radiuskopf aufgefädelt und in die korrekte Position geschoben wird (『 Abb. 11).

Prinzipiell gilt, bei Kindern „eher geschlossen als offen vorgehen“. Die anatomische Stellung muss nicht um jeden Preis erzwungen werden, Richtlinien für eine akzeptable Reposition sind die in - Tab. 2 angegebenen Entscheidungshilfen für die Frage einer operativen vs. konservativen Therapie. Nach 3 Versuchen der geschlossenen Reposition sollten die offenen Reposition und Osteosynthese erfolgen. 
Tab. 2 Altersrichtwerte für die akzeptable Dislokation

\begin{tabular}{|c|c|c|}
\hline Alter (Jahre) & Abkippung & Schaftbreite \\
\hline$<5$ & $60^{\circ}$ & $1 / 2$ des Schafts \\
\hline $5-10$ & $40^{\circ}$ & $1 / 3$ des Schafts \\
\hline $11-14$ & $20^{\circ}$ & $1 / 3$ des Schafts \\
\hline$>14$ & $10^{\circ}$ & $1 / 5$ des Schafts \\
\hline
\end{tabular}

\section{Komplikation}

In jeden Fall muss nach Begleitverletzungen gesucht werden, hier stehen Monteggia-Verletzungen (insbesondere BadoKlasse-IV-Monteggia-Verletzung: komplette Unterarmfraktur mit Ulnafraktur und proximaler Radiusfraktur) im Vordergrund. Darüber hinaus muss auch eine Pronatio Dolorosa (traumatische Radiusköpfchensubluxation bei forciertem Zug am ausgestreckten Arm des Kindes) ausgeschlossen werden.

\section{Korrespondenzadresse \\ PD Dr. P. Biberthaler}

Chirurgische Klinik, Innenstadt, Ludwig-Maximilians-Universität München, Nußbaumstraße 20, 80336 München Peter.Biberthaler@med.uni-muenchen.de

Interessenkonflikt. Der korrespondierende Autor gibt an, dass kein Interessenkonflikt besteht.

\section{Literatur}

1. Ashwood N, Bain GI, Unni R (2004) Management of Mason type-III radial head fractures with a titanium prosthesis, ligament repair, and early mobilization. J Bone Joint Surg Am 86-A(2):274-280

2. Bain Gl, Ashwood N, Baird R, Unni R (2005) Management of Mason type-III radial head fractures with a titanium prosthesis, ligament repair, and early mobilization. Surgical technique. J Bone Joint Surg Am [Suppl 1] 87(Pt 1):136-147

3. Beingessner DM, Dunning CE, Gordon KD et al (2004) The effect of radial head excision and arthroplasty on elbow kinematics and stability. J Bone Joint Surg Am 86-A(8):1730-1739

4. Boulas HJ, Morrey BF (1998) Biomechanical evaluation of the elbow following radial head fracture. Comparison of open reduction and internal fixation vs. excision, silastic replacement, and non-operative management. Chir Main 17(4):314-320

5. Broberg MA, Morrey BF (1987) Results of treatment of fracture-dislocations of the elbow. Clin Orthop 216:109-119

6. Davidson PA, Moseley JB Jr, Tullos HS (1993) Radial head fracture. A potentially complex injury. Clin Orthop 297:224-230

7. Furry KL, Clinkscales CM (1998) Comminuted fractures of the radial head. Arthroplasty versus internal fixation. Clin Orthop 353:40-52
8. Greenspan A, Norman A (1982) The radial head, capitellum view: useful technique in elbow trauma. AJR Am J Roentgenol 138(6):1186-1188

9. Harrington IJ, Tountas AA (1981) Replacement of the radial head in the treatment of unstable elbow fractures. Injury 12(5):405-412

10. Harrington IJ, Sekyi-Otu A, Barrington TW et al (2001) The functional outcome with metallic radial head implants in the treatment of unstable elbow fractures: a long-term review. J Trauma 50(1):4652

11. Herbertsson $P$, Josefsson $P O$, Hasserius R et al (2004) Fractures of the radial head and neck treated with radial head excision. J Bone Joint Surg Am 86-A(9):1925-1930

12. Herbertsson $P$, Josefsson $P O$, Hasserius $R$ et al (2004) Uncomplicated Mason type-II and III fractures of the radial head and neck in adults. A longterm follow-up study. J Bone Joint Surg Am 86A(3):569-574

13. Holmenschlager F, Halm JP, Winckler S (2002) Fresh fractures of the radial head: results with the Judet prosthesis. Rev Chir Orthop Reparatrice Appar Mot 88(4):387-397

14. Hotchkiss RN (1997) Displaced fractures of the radial head: internal fixation or excision? J Am Acad Orthop Surg 5(1):1-10

15. Hotchkiss RN, Weiland AJ (1987) Valgus stability of the elbow. J Orthop Res 5(3):372-377

16. Ikeda M, Oka Y (2000) Function after early radial head resection for fracture: a retrospective evaluation of 15 patients followed for 3-18 years. Acta Orthop Scand 71(2):191-194

17. Ikeda M, Yamashina Y, Kamimoto M, Oka Y (2003) Open reduction and internal fixation of comminuted fractures of the radial head using low-profile mini-plates. J Bone Joint Surg Br 85(7):1040-1044

18. Ikeda M, Sugiyama K, Kang C et al (2005) Comminuted fractures of the radial head. Comparison of resection and internal fixation. J Bone Joint Surg Am 87(1):76-84

19. Jensen SL, Olsen BS, Tyrdal S et al (2005) Elbow joint laxity after experimental radial head excision and lateral collateral ligament rupture: efficacy of prosthetic replacement and ligament repair. J Shoulder Elbow Surg 14(1):78-84

20. Johnson JA, Beingessner DM, Gordon KD et al (2005) Kinematics and stability of the fractured and implant-reconstructed radial head. J Shoulder Elbow Surg [Suppl 1S] 14:195S-201S

21. King GJ (2004) Management of comminuted radial head fractures with replacement arthroplasty. Hand Clin 20(4):429-441

22. King GJ, Evans DC, Kellam JF (1991) Open reduction and internal fixation of radial head fractures. J Orthop Trauma 5(1):21-28

23. Morrey BF, Tanaka S, An KN (1991) Valgus stability of the elbow. A definition of primary and secondary constraints. Clin Orthop 265:187-195

24. Pomianowski S, Morrey BF, Neale PG et al (2001) Contribution of monoblock and bipolar radial head prostheses to valgus stability of the elbow. J Bone Joint Surg Am 83-A(12):1829-1834

25. Ring D (2004) Load-sharing at the wrist following radial head replacement with a metal implant. A cadaveric study. J Bone Joint Surg Am 86-A(11):2569-2570

26. Ring D, Quintero J, Jupiter JB (2002) Open reduction and internal fixation of fractures of the radial head. J Bone Joint Surg Am 84-A(10):1811-1815

27. Rozental TD, Beredjiklian PK, Bozentka DJ (2003) Longitudinal radioulnar dissociation. J Am Acad Orthop Surg 11(1):68-73
28. Smith GR, Hotchkiss RN (1996) Radial head and neck fractures: anatomic guidelines for proper placement of internal fixation. J Shoulder Elbow Surg 5(2 Pt 1):113-117

29. Stein DA, Patel R, Egol KA et al (2003) Prevention of heterotopic ossification at the elbow following trauma using radiation therapy. Bull Hosp Jt Dis 61(3-4):151-154

30. Tejwani NC, Mehta H (2007) Fractures of the radial head and neck: current concepts in management. J Am Acad Orthop Surg 15(7):380-387

31. Van Glabbeek F, Van Riet RP, Baumfeld JA et al (2004) Detrimental effects of overstuffing or understuffing with a radial head replacement in the medial collateral-ligament deficient elbow. J Bone Joint Surg Am 86-A(12):2629-2635

32. Van Glabbeek F, Van Riet RP, Baumfeld JA et al (2005) The kinematic importance of radial neck length in radial head replacement. Med Eng Phys 27(4):336-342 